\title{
Editorial
}

\section{The Anti-Diabetic Drug Metformin from the Neuropathy Perspective}

\author{
Jong Chul Won \\ Division of Endocrinology and Metabolism, Department of Internal Medicine, Cardiovascular and Metabolic Disease Center, Inje University Sanggye Paik \\ Hospital, Inje University College of Medicine, Seoul, Korea
}

Diabetic peripheral neuropathy (DPN) is the most prevalent complication in patients with diabetes and usually manifests early during diabetes, decreasing quality of life and sleep [1]. Early preventive measures and management of DPN is essential in patients with diabetes; however, there are still unmet needs in clinical practice largely caused by a lack of mechanism- or pathogenetic-based therapies. Management of DPN is based on symptomatic and pathogenetic treatment [2]. While rigorous control of hyperglycemia is a proven therapeutic option in patients with type 1 diabetes mellitus (T1DM), it is less established in those with type 2 diabetes mellitus (T2DM). Other disease-modifying pathogenetic treatments including $\alpha$-lipoic acid (ALA) or $\gamma$-linolenic acid has been suggested, but more comprehensive clinical trials are needed to confirm their efficacy and safety. Alternately a number of symptomatic treatments to relieve pain or discomfort are recommended in clinical guidelines, including antidepressants such as amitriptyline and duloxetine, $\gamma$-aminobutyric acid analogues gabapentin and pregabalin, opioids, and topical agents such as capsaicin, which have limited efficacy for pain control in T1DM or T2DM.

In this issue of the Diabetes and Metabolism Journal, Kim et al. [3] investigated the effects of metformin on sensory tests including sensitivity to a von Frey filament, paw-licking and tail flick response to a hot plate, as well as a Randall-Selittometer to assess pressure threshold, and comparison analysis of neuronal quantification in streptozotocin/high-fat diet-induced diabetic rats. In addition, they compared plasma levels of vitamin B12, glutathione, and tumor necrosis factor $\alpha$ (TNF- $\alpha$ ). In this ex- periment, the authors treated rats with metformin at $100 \mathrm{mg} /$ $\mathrm{kg}$ /day to minimize the glucose lowering effect, and they administered ALA $(0.5 \%, 100 \mathrm{mg} / \mathrm{kg} /$ day $)$ as an active control. They found 12-week treatment with metformin attenuated the hypersensitivity reaction to a von Frey filament (tactile allodynia) in diabetic rats, and these effects were maintained with 24-week treatment. However, thermal hyperalgesia (paw and tail response to hot plates) was not attenuated by metformin at 12- and 24-week time points. Effects of metformin treatment on the preservation of tactile and pressure responses were comparable to treatment with ALA at 24 weeks. They also found a neuroprotective effect of metformin by measuring intraepidermal nerve fiber density (IENFD) and axon diameter in cutaneous peripheral and sciatic nerves, respectively. In addition, they found that vitamin B12 levels were not decreased by metformin treatment compared to normal and diabetic rats while TNF- $\alpha$ levels were decreased by treatment with metformin or ALA compared to diabetic rats. Finally, the authors proposed that the neuroprotective benefit of metformin might be associated with anti-inflammatory properties by reducing proinflammatory TNF- $\alpha$ levels, which are independent of glucoselowering effects. These results suggest that 24-week treatment with metformin initiated early in the course of T1DM attenuate the hypersensitivity resulting from axonal degeneration by decreasing systemic inflammation related to hyperglycemia; thus, metformin has therapeutic potential to prevent or delay hyperglycemia-induced peripheral nerve damage.

Although metformin, a widely used anti-diabetic drug that
Corresponding author: Jong Chul Won (iD https://orcid.org/0000-0002-2219-4083 Department of Internal Medicine, Cardiovascular and Metabolic Disease Center, Inje University Sanggye Paik Hospital, Inje University College of Medicine, 1342 Dongil-ro, Nowon-gu, Seoul 01757, Korea

E-mail:drwonjc@gmail.com
This is an Open Access article distributed under the terms of the Creative Commons Attribution Non-Commercial License (https://creativecommons.org/licenses/by-nc/4.0/) which permits unrestricted non-commercial use, distribution, and reproduction in any medium, provided the original work is properly cited. 
activates adenosine monophosphate-activated protein kinase (AMPK) [4], has beneficial effects in patients with T2DM from a glucocentric viewpoint, it is reported to reduce vitamin B12 absorption in the terminal ileum [5], and there are concerns about the development or worsening of DPN in patients with T2DM [6]. However, controversy remains because vitamin B12 deficiency-associated neuropathy is indistinguishable from DPN in patients with T2DM and there are many confounding factors, including age, study setting, diabetes duration, and dose and duration of metformin in clinical studies. Therefore, no direct link between metformin use and DPN has been established $[6,7]$. On the other hand, recent findings with animal models showed that metformin has neuroprotective effects through AMPK activation and inhibition of mammalian target of rapamycin resulting in inactivation of extracellular signalregulated kinases, which increases the excitability of nociceptors [8], and metformin also has a positive central effects on nociceptors, reducing thermal and mechanical hypersensitivity [9]. In line with the current study, previous experiments found that metformin prevented atrophy of myelinated axons and reduced expression of inflammatory mediators (interleukin-1 $\beta$, inducible nitric oxide synthase and nitric oxide); these effects were more prominent after treatment with $200 \mathrm{mg}$ compared to $100 \mathrm{mg}$ of metformin in streptozotocin-induced diabetic mice [10].

In this issue, Kim et al. [3] demonstrated that metformin treatment preserved tactile allodynia and peripheral nerve integrity (IENFD) without glucose-lowering effects or vitamin B12 deficiency in a murine model of T1DM. Because there are different mechanisms underlying the course of DPN between T1DM and T2DM, the results of this experiment need to be confirmed. In addition, it remains unclear whether hypersensitivity results from axonal degeneration or demyelination of small nerve fibers, and how metformin or ALA prevented decreases in axon diameter but not myelin sheath thickness in this experiment. Therefore, future studies need to establish the mechanisms by which metformin exerts neuroprotective effects on axonal degeneration occurring in the early stage of diabetes in addition to glucocentric and vascular benefits in $\mathrm{T} 2 \mathrm{DM}$, in order to develop the novel therapeutic interventions.

\section{CONFLICTS OF INTEREST}

No potential conflict of interest relevant to this article was reported.

\section{REFERENCES}

1. Won J, Kwon H, Kim C, Lee J, Park T, Ko K, Cha B. Prevalence and clinical characteristics of diabetic peripheral neuropathy in hospital patients with type 2 diabetes in Korea. Diabetic Med 2012;29:e290-6.

2. American Diabetes Association. 11. Microvascular complications and foot care: standards of medical care in diabetes-2020. Diabetes Care 2020;43(Suppl 1):S135-51.

3. Kim SH, Park TS, Jin HY. Metformin preserves peripheral nerve damage with comparable effects to alpha lipoic acid in streptozotocin/high-fat diet induced diabetic rats. Diabetes Metab J 2020;44:842-53.

4. Cryer PE. Comment on: Nathan DM, Buse JB, Davidson MB et al (2006) Management of hyperglycaemia in type 2 diabetes: a consensus algorithm for the initiation and adjustment of therapy. A consensus statement from the American Diabetes Association and the European Association for the Study of Diabetes. Diabetologia 49:1711-1721. Diabetologia 2007;50:2224.

5. Tomkin GH. Malabsorption of vitamin B12 in diabetic patients treated with phenformin: a comparison with metformin. $\mathrm{Br}$ Med J 1973;3:673-5.

6. Wile DJ, Toth C. Association of metformin, elevated homocysteine, and methylmalonic acid levels and clinically worsened diabetic peripheral neuropathy. Diabetes Care 2010;33:156-61.

7. Ahmed MA, Muntingh G, Rheeder P. Vitamin B12 deficiency in metformin-treated type-2 diabetes patients, prevalence and association with peripheral neuropathy. BMC Pharmacol Toxicol 2016;17:44.

8. Ji RR, Gereau RW 4th, Malcangio M, Strichartz GR. MAP kinase and pain. Brain Res Rev 2009;60:135-48.

9. Shiers S, Pradhan G, Mwirigi J, Mejia G, Ahmad A, Kroener S, Price T. Neuropathic pain creates an enduring prefrontal cortex dysfunction corrected by the type II diabetic drug metformin but not by gabapentin. J Neurosci 2018;38:7337-50.

10. Los DB, de Oliveira WH, Duarte-Silva E, Sougey WWD, de Freitas EdSR, de Oliveira AGV, Braga CF, de Franca MER, da Rocha Araujo SM, Rodrigues GB, Rocha SWS, Peixoto CA, de Moraes SRA. Preventive role of metformin on peripheral neuropathy induced by diabetes. Int Immunopharmacol 2019;74: 105672. 